\title{
A Clove-Hitch Suture Method for Small-Caliber Tendon Ends
}

\author{
Edward Abraham, MD, Alan League, MD, Farid Amirouche, PhD, and Nikhil Kulkarni, MS Eng \\ Investigation performed at the Department of Orthopaedics, University of Illinois College of Medicine, \\ and the Department of Mechanical and Industrial Engineering, University of Illinois at Chicago, Chicago, Illinois
}

\begin{abstract}
Background: The choices of suture methods used to secure the end of a small-caliber tendon with a combination of suture materials passing through and looping around the tendon are not well documented. A secure stitchtendon unit may be an important factor in preserving muscle function, by facilitating healing without gapping or failure.
\end{abstract}

Methods: Five types of suture methods utilizing four or fewer passes through the tendon end were mechanically tested on 178 canine flexor digitorum profundus tendons. An Instron apparatus was used to test the load to failure.

Results: The single and double clove-hitch suture methods demonstrated improved repair strength when compared with the three other methods studied in this small-caliber canine tendon model.

Conclusions: The single and double clove-hitch suture method better secured the end of a small-caliber tendon compared with other methods.

Clinical Relevance: The single (pull-out) and double (non-pull-out) clove-hitch suture methods are reliable alternatives for the repair of small-caliber tendons.

$\mathrm{T}$

he secure repair of soft tissue to bone, such as with tendon repair or tendon transfer, is essential to facilitate physiologic healing. At our institution, the senior author (E.A.) has been using both the single and the double clove-hitch suture method to secure small-caliber tendons and ligaments for over three decades. A search of the Englishlanguage literature failed to identify any publications comparing the single (pull-out) and double (non-pull-out) clove-hitch suture methods with others. We hypothesized that the clovehitch suture methods offer pull-out strength that is superior to that provided by other simple suture methods used to secure small-caliber tendon ends. The aim of this biomechanical study was to compare the pull-out strength provided by the single and double clove-hitch suture methods with that offered by three other suture methods in an in vitro canine flexor tendon model.

\section{Materials and Methods}

ne hundred and seventy-eight flexor digitorum profundus tendons were harvested from the hind paws of twenty adult mongrel dogs weighing between 20 and $25 \mathrm{~kg}$. The animals were killed for other purposes that did not involve the hind legs. The original research on the animals had received approval by the Animal Care Committee. The average width of the tendons was $4.3 \mathrm{~mm}$, with a range from 3.5 to $4.5 \mathrm{~mm}$, and the average depth was $2.6 \mathrm{~mm}$, with a range from 2.0 to $3.0 \mathrm{~mm}$. These measurements were obtained by using a tumor meter calibrated to $0.1 \mathrm{~mm}$ (U.S. Patent 423960; BHS International, Crofton, Maryland) and a magnifying glass apparatus. The specimens were wrapped in normal saline solution-soaked gauze and then placed in plastic zip lock bags. They were stored in a refrigerator at $2^{\circ} \mathrm{C}$ for up to three days. Only $4 \%$ of the specimens were stored in a freezer at $-70^{\circ} \mathrm{C}$ for up to seven days. These tendons were thawed over a twenty-four-hour period in a refrigerator and then were warmed to $37^{\circ} \mathrm{C}$ prior to biomechanical testing.

Five different suture techniques were compared. The same suture material, size 2-0 braided Ethibond (Ethicon, Somerville, New Jersey), was used in all constructs. In preliminary trials, a weaker-gauge suture material

Disclosure: None of the authors received payments or services, either directly or indirectly (i.e., via his or her institution), from a third party in support of any aspect of this work. None of the authors, or their institution(s), have had any financial relationship, in the thirty-six months prior to submission of this work, with any entity in the biomedical arena that could be perceived to influence or have the potential to influence what is written in this work. Also, no author has had any other relationships, or has engaged in any other activities, that could be perceived to influence or have the potential to influence what is written in this work. The complete Disclosures of Potential Conflicts of Interest submitted by authors are always provided with the online version of the article. 
The Journal of Bone \& Joint Surgery · Jbjs.org Volume 94-A · Number $14 \cdot$ July 18,2012
A Clove-Hitch Suture Method for Small-Caliber

TENDON ENDS
A

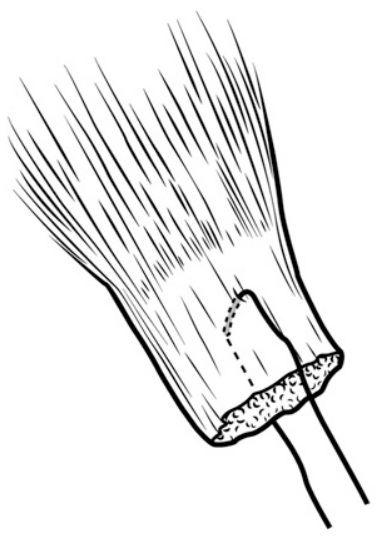

C

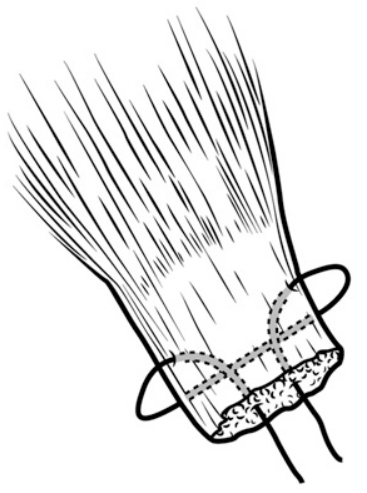

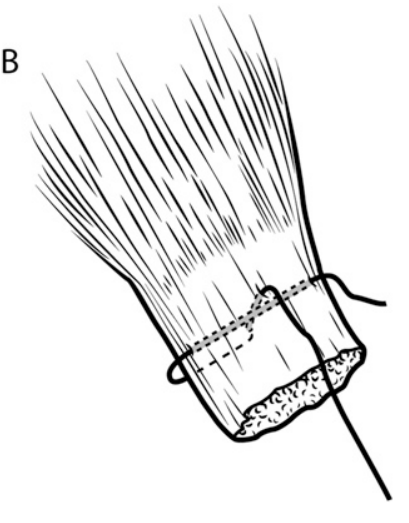

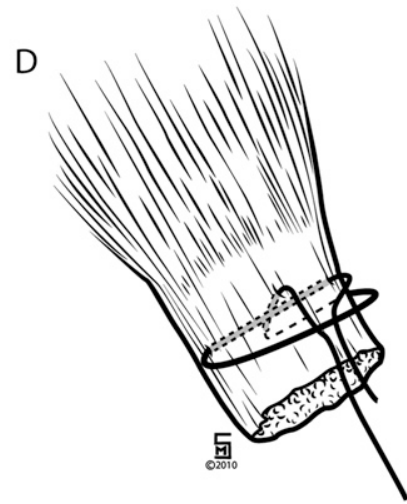

Fig. 1

The five suture methods tested. A: Single pass across the tendon. B: Double pass across the tendon with the passes at $90^{\circ}$ to one another. C: Modified Kessler stitch. D: Single clove-hitch suture method. Suture method E, the double clove-hitch method, is the same as method D except that it is repeated adjacently to the first one with the same suture material (see Fig. 2, E). (Reproduced with permission of Melissa Sisk.)

such as 3-0 braided Ethibond ruptured before failure of the suture-tendon unit. The five different repair methods were labeled as A (a single pass across the tendon; thirty-three tendons), B (a double pass across the tendon with the passes at $90^{\circ}$ to each other; thirty-three tendons), C (a modified Kessler stitch $^{1}$; thirty-three tendons), D (the single clove-hitch suture method; thirty-three tendons), or E (the double clove-hitch suture method; twentythree tendons) (Fig. 1 and Fig. 2, E). All stitches were placed $1 \mathrm{~cm}$ proximal from the distal end of each tendon. The tendons were divided randomly into five test groups. All repairs were performed by an orthopaedic resident. The first three suture methods, which utilized three or fewer passes through the tendon, were chosen on the basis of our clinical practice and anecdotal observations of other surgeons in the community.

The single (pull-out) clove-hitch suture method is demonstrated in Figure 2 . First, the tapered needle was passed horizontally across the tendon (Fig. 2, A). The second pass of the needle was vertical, or $90^{\circ}$, and just proximal to the first (Fig. 2, B). The free ends of the suture material were placed through the suture loop (Fig. 2, C). The two free ends were pulled tight so as to secure about three-quarters of the tendon thickness (Fig. 2, D). In this study, all of the suturing was done by a right-handed individual, so that the needle went from right to left across the tendon in the first step; in the second step, the needle traveled from anterior to posterior.

The double clove-hitch suture method consisted of two single clovehitch suture methods performed by using the same suture strand (Fig. 2, E).

After completion of the first clove-hitch suture as described above, the second clove-hitch suture was performed 1 to $5 \mathrm{~mm}$ proximal or distal to the first one. The second suture was then tightened by pulling the one sliding end of the suture material.

The breaking strength associated with the five suture methods was measured with an Instron 5569 materials testing machine (Norwood, Massachusetts). The instrument had a loading capacity of 2 to $600 \mathrm{~kg}$. The tendon was loaded vertically in the Instron machine with the suture material tied securely to a metal hook and the other end of the tendon fixed between the flat plates of an immobile metal clamp. The average distance between the hook and clamp was $15 \mathrm{~cm}$.

Tension was applied through the hook at a rate of $20 \mathrm{~mm}$ per minute, until complete rupture of the stitch-tendon unit occurred. The force needed to produce the rupture was measured in newtons $(\mathrm{N})$ and recorded in the form of a force versus displacement curve. Before each specimen was tested, the load and displacement were changed to start from no load and zero displacement.

\section{Source of Funding}

No external funding was received for this study.

\section{Statistical Analysis}

Statistical comparisons among the five suture types were done with use of the Statistical Package for Social Sciences (version 17.0; SPSS, Chicago, Illinois). The level of significance was set at $\mathrm{p} \leq 0.05$ for all analyses. Analysis of variance (ANOVA) was used for testing the mean values of all five stitch types. Bonferroni and Dunnett-T3 testing were used to analyze the homogeneous and heterogeneous nature of the variances, respectively. Merlin software (Merlin Technical Solutions, Greenwood Village, Colorado) was used to collect the data from each specimen. The initial sample size was chosen by convenience. With use of the ANOVA procedure, the means were viewed as significantly different if $\alpha$ was $<0.01$. The mean square error of 41.6 was obtained by the same procedure, for $150^{\circ}$ of freedom. The sample-size estimates were obtained with use of a non-central $\mathrm{F}$ distribution and a power of 0.99 .

\section{Results}

The force required to cause a failure of the stitch-tendon unit created with either the single or the double clovehitch suture method was significantly greater than the force required after use of any of the other three suture methods ( $\mathrm{p} \leq$ 0.05 ) (Fig. 3). The average breaking strength was $11.06 \mathrm{~N}$ in Group A (single pass), $22.98 \mathrm{~N}$ in Group B (two passes at $90^{\circ}$ to each other), $32.68 \mathrm{~N}$ in Group C (modified Kessler suture), $39.24 \mathrm{~N}$ in Group D (single clove-hitch suture), and $51.71 \mathrm{~N}$ in Group E (double clove-hitch suture). The double clovehitch suture method resulted in a $32 \%$ higher strength as compared with the single clove-hitch suture method ( $\mathrm{p} \leq$ 0.05 ). Although the modified Kessler suture method involved three passes through the tendon, it created a construct that was significantly weaker than that provided by the single clove-hitch suture method, which consisted of two passes through the tendon with a loop. The load to failure of each stitch-tendon unit tested in Group A was less than that of any of those tested in Groups C, D, and E. Also, each of the tendons tested in Group B failed at a load lower than that required for failure of any of the tendons examined in Group E. The most common mode of repair site failure was suture pullout from the tendon substance (in Groups A through D) or 


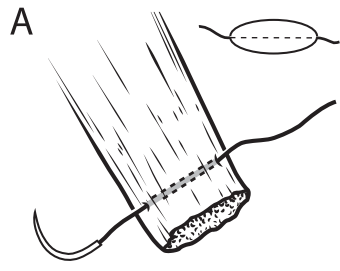

Tendon

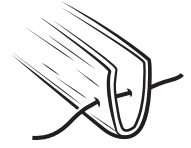

Fascia
B

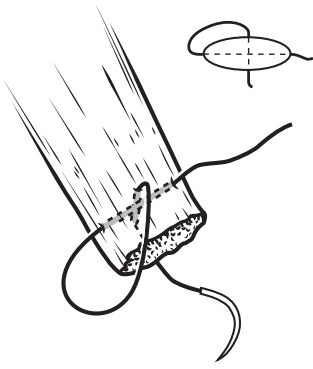

C

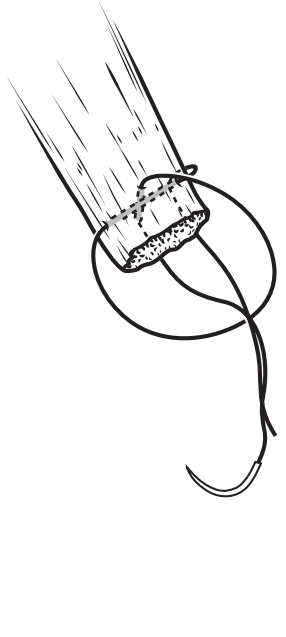

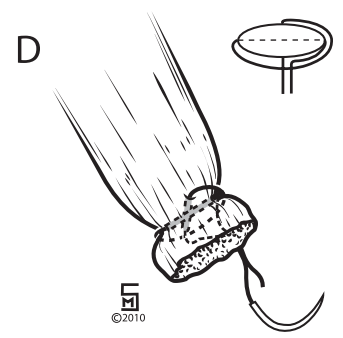

$\mathrm{E}$

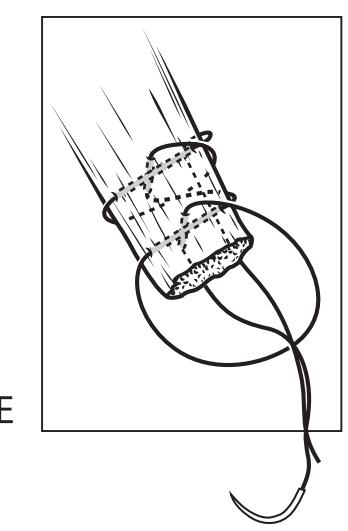

Fig. 2

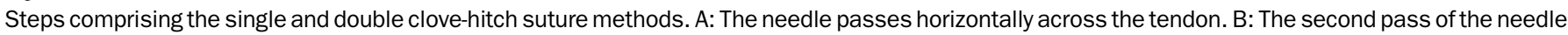

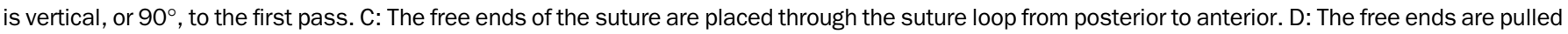

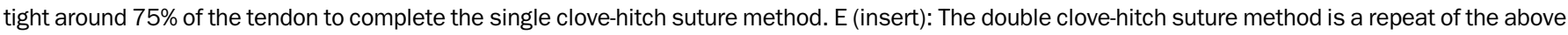

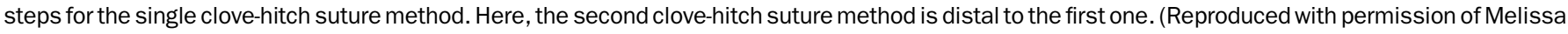
Sisk.)

tearing away (in Group E). Tearing away of parts of the tendon tissue was seen more frequently with the double clove-hitch suture method. Breakage of the suture was not observed in any specimen.

\section{Discussion}

$\mathbf{B}$ oth the single and the double clove-hitch suture method B provided constructs that were stronger than those created with the three other suture methods in this in vitro canine small-caliber tendon model $(\mathrm{p} \leq 0.05)$. Additionally, the construct created with the double clove-hitch suture method was significantly stronger than that created with the single clovehitch suture method or with any of the other three suture methods tested ( $\mathrm{p} \leq 0.05)$. No other suture methods were tested in this study. In a comparable biomechanical study, the modified Kessler suture method was compared with other types of repairs ${ }^{2}$. The average breaking strength of the tendonto-tendon stitch unit created with the modified Kessler suture technique in that study was between 20 and $25 \mathrm{~N}$ (average, 23 $\mathrm{N}$ ), which was comparable with the 11 to $39 \mathrm{~N}$ (average, $26 \mathrm{~N}$ ) in the present study. However, the load-to-failure values in both studies were significantly lower than the $301 \mathrm{~N}( \pm 24 \mathrm{~N})$ with the Krackow stitch, which was tested on larger-caliber porcine Achilles tendons ${ }^{3}$. We did not compare the double clove-hitch suture method with any non-pull-out suture methods for small-caliber tendons. Both the single clove-hitch suture method (D) and the suture method involving the double pass across the tendon (B) penetrate the tendon twice in an identical fashion, but adding the suture loop step to suture method B to make the single clove-hitch suture method created a significantly stronger construct. Also, the addition of a second clove-hitch suture method adjacent to the first one, to make the double clove-hitch suture method (E), significantly strengthened the stitch-tendon unit.

The double clove-hitch suture is not recommended for use as a pull-out suture because of the multiple passes of suture material through the tendon. Also, performing the second pass of the suture strand $5 \mathrm{~mm}$ proximal to the first pass in the double clove-hitch suture method was our preferred technique because better-quality tissue was usually available in that area. We did not compare the mechanical effects of placing the second pass $5 \mathrm{~mm}$ proximal to the first pass with the effects of placing it $5 \mathrm{~mm}$ distal to the first pass.

In 1964, Sharrard reported the results of posterior iliopsoas tendon transplantation for paralytic dislocation of the hip. He wrote: "A strong silk suture is attached by clove-hitch to the fragment of lesser trochanter and to the iliopsoas tendon." The iliopsoas tendon was passed through a bone tunnel into the greater trochanter and secured to adjacent soft tissue. He did not give a rationale for naming the knot clove-hitch. In his original description and in the current technique, the suture material passes through and around the tissue rather than just around it. While working as a senior registrar on Mr. Sharrard's service in 1975, the senior author of the current study learned to use the 
The Journal of Bone \& Joint Surgery $\cdot$ JBJS.org Volume 94-A · Number $14 \cdot$ July 18,2012

A Clove-Hitch Suture Method for Small-Caliber TENDON ENDS

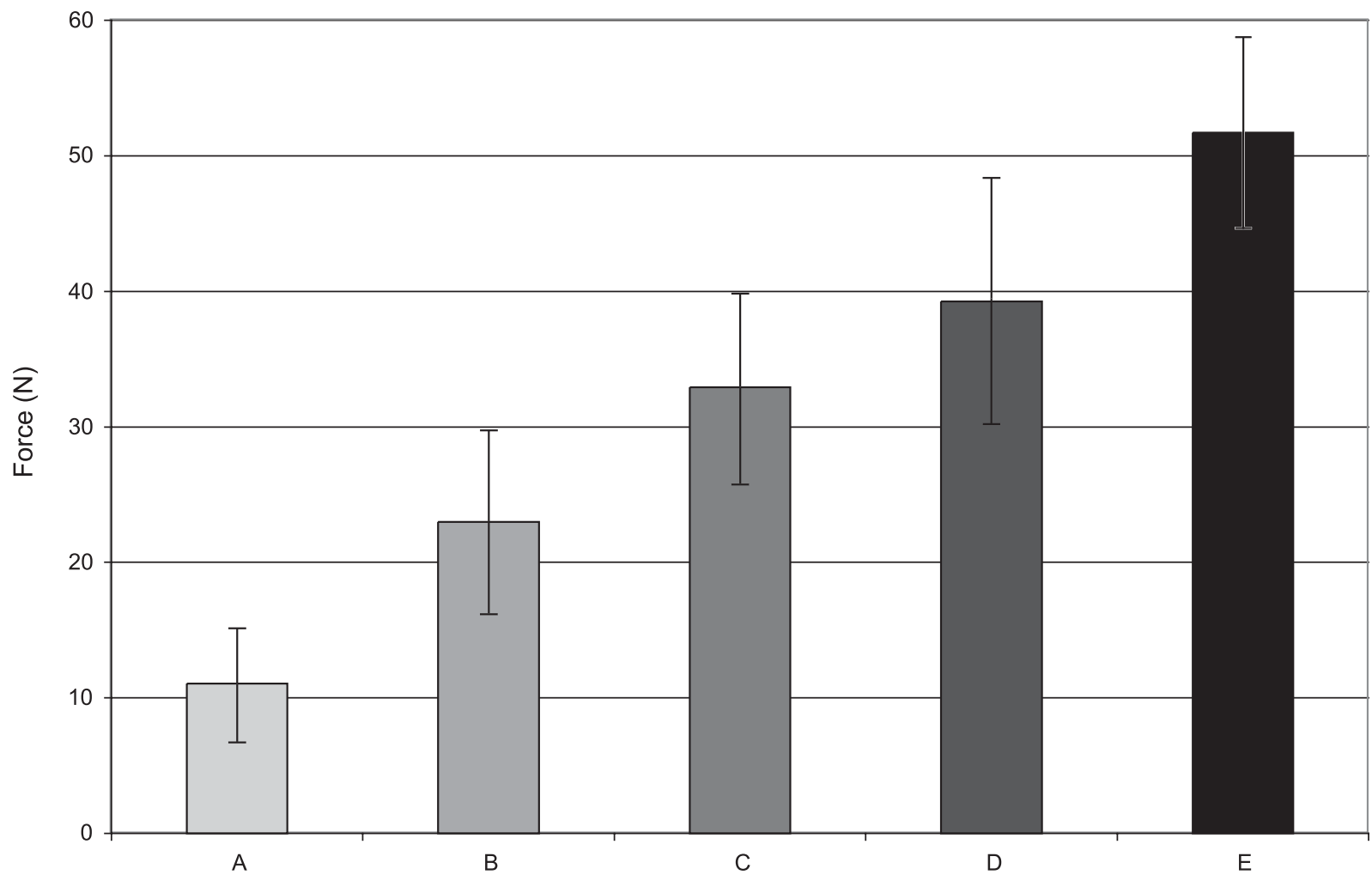

Fig. 3

Comparison of the average breaking strength of the different stitch-tendon units. A: Single pass across the tendon. B: Double pass across the tendon. C: Modified Kessler stitch (three passes). D: Single clove-hitch suture method (two passes). E: Double clove-hitch suture method (four passes). The I bars indicate the standard deviation.

clove-hitch suture method. Since that time, it has been utilized to secure small-caliber tendons, ligaments, and fascial strips. For example, we commonly utilize the pull-out single clove-hitch suture method for a tibialis anterior tendon transfer to a cuboid osseous tunnel for treatment of patients with residual congenital clubfoot deformity.

A unique benefit of the single clove-hitch suture construct in comparison with the Krackow suture construct is that it can be removed at a later date by cutting one suture limb at skin level and then pulling the other suture limb. This pull-out feature of the suture method is not possible with suture methods in which multiple fixation points are used on the tendon ${ }^{5-7}$. The single clove-hitch suture method can be used to secure a flat tendon or a narrow fascial strip by weaving the horizontal limb of suture strand in and out of the tissue.

Limitations of the current study include (1) biomechanical testing was carried out in an in vitro setting on canine tendons, which may have properties that differ from those of a human tendon; (2) the physiology of tendon healing at the repair site following the repairs could not be assessed; (3) the potential effect of cyclic loading on the load-to-failure testing was not studied; (4) limited suture comparisons were carried out; (5) the potential strangulation effects of the clove-hitch suture at the repair site were not studied; (6) the effects of possible variations of placement of the second clove-hitch suture construct on the biologic processes of tendon breaking was not evaluated; and (7) author advocacy is an inherent bias of the study.

In conclusion, the single and double clove-hitch suture methods were found to result in superior load-to-failure strength when compared with three commonly used pull-out suture methods in this in vitro canine tendon repair model.

Edward Abraham, MD

Alan League, MD

Department of Orthopaedics,

University osf Illinois College of Medicine,

835 South Wolcott Avenue,

E270, Chicago, IL 60612.

E-mail address for E. Abraham: Eda@uic.edu.

E-mail address for A. League: Leag0002@yahoo.com

Farid Amirouche, $\mathrm{PhD}$

Nikhil Kulkarni, MS Eng

Department of Mechanical and Industrial Engineering,

University of Illinois at Chicago,

2039 Engineering Research Facility,

842 West Taylor Street, Chicago, IL 60607.

E-mail address for F. Amirouche: amirouch@uic.edu.

E-mail address for N. Kulkarni: nikhil80@gmail.com 


\section{References}

1. Kessler I. The "grasping" technique for tendon repair. Hand. 1973 Oct;5(3): 253-5.

2. Momose T, Amadio PC, Zhao C, Zobitz ME, Couvreur PJ, An KN. Suture techniques with high breaking strength and low gliding resistance: experiments in the dog flexor digitorum profundus tendon. Acta Orthop Scand. 2001 Dec; 72(6):635-41.

3. White KL, Camire LM, Parks BG, Corey WS, Hinton RY. Krackow locking stitch versus locking premanufactured loop stitch for soft-tissue fixation: a biomechanical study. Arthroscopy. 2010 Dec;26(12):1662-6.
4. Sharrard WJ. Posterior iliopsoas transplantation in the treatment of paralytic dislocation of the hip. J Bone Joint Surg Br. 1964 Aug;46:426-44

5. Krackow KA, Thomas SC, Jones LC. A new stitch for ligament-tendon fixation. Brief note. J Bone Joint Surg Am. 1986 Jun;68(5):764-6.

6. Krackow KA, Cohn BT. A new technique for passing tendon through bone. Brief note. J Bone Joint Surg Am. 1987 Jul;69(6):922-4.

7. Thompson $\mathrm{GH}$, Hoyen $\mathrm{HA}$, Barthel T. Tibialis anterior tendon transfer after clubfoot surgery. Clin Orthop Relat Res. 2009 May;467(5):1306-13. Epub 2009 Feb 26. 